\title{
Analysis of Thickness Vibration Frequencies of FBAR as Layered Structures with Piezoelectric Plates
}

\author{
Yurun $\mathrm{Chen}^{a}$, JiAnsong LiU ${ }^{a}$, Ji WAnG ${ }^{a, b, *}$, \\ Tingfeng MA $\mathrm{MA}^{a, b}$, Jianke DU ${ }^{a, b}$ And Honglang $\mathrm{LI}^{c}$ \\ ${ }^{a}$ Piezoelectric Device Laboratory, School of Mechanical Engineering 83 Mechanics, \\ Ningbo University, 818 Fenghua Road, Ningbo, 315211 Zhejiang, China \\ ${ }^{b}$ TXC-NBU Joint Center of Research, School of Mechanical Engineering $\& 3$ Mechanics, \\ Ningbo University, 818 Fenghua Road, Ningbo, 315211 Zhejiang, China \\ ${ }^{c}$ National Center for Nanoscience and Technology, \\ 11 Beiyitiao Road, Zhongguancun, 100190 Beijing, China
}

Received: 05.07.2021 \& Accepted: 29.07.2021

Doi: 10.12693/APhysPolA.140.105

*e-mail: wangji@nbu.edu.cn

\begin{abstract}
Film bulk acoustic resonators as a newer generation of acoustic wave resonators have been a proper replacement of traditional resonators for frequency control and sensor applications with a fast adoption in mobile communication and other major consumer electronics. Extensive research work on the materials, processing, structure, and design has contributed to the phenomenal growth of film bulk acoustic resonators. As a procedure for the selection of optimal design parameters, we started from the layered structures of film bulk acoustic resonators with a one-dimensional model, i.e., only considering the thickness of resonators for the calculation of vibration frequencies in order to support the resonator design. The resonant frequency of thickness-extension mode - a key parameter of resonators — was obtained through the consideration of the elastic and piezoelectric properties of each layer in the resonators structure. It has been proven through numerical examples that in this way we gain an effective approach to the accurate determination of resonant frequency. Clearly, the method based on film bulk acoustic resonators is also helpful for the early stage of product design in the selection of some essential parameters and later combinations for device upgrades.
\end{abstract}

topics: FBAR, wave, vibration, frequency

\section{Introduction}

Thin film bulk acoustic resonators (FBAR) [1-5] are a newer generation of products in piezoelectric acoustic wave device industry, which is vigorously replacing some applications of surface acoustic wave (SAW) resonators $[6,7]$ and quartz crystal resonators [8] due to its higher resonance frequency and smaller size. FBAR have the typical layered structure, that is a piezoelectric thin film is sandwiched by metal electrodes on the Bragg reflection layers and the substrate [9-11]. All these developments of a new type of piezoelectric acoustic wave resonators, of course, are based on the precise design and fabrication. These resonators are typical thin film structures with larger quantities of layers. The utilization of piezoelectric thin films for the generation of acoustic waves with higher frequency poses another challenge, because it requires more accurate analysis and design to realize devices functions in higher frequency.
The general methods for the analysis of propagation of acoustic waves in FBAR, which are needed for the initial design of piezoelectric resonators, provide essential solutions of vibration frequency and deformation. Many methods for the analysis of layered piezoelectric devices for acoustic wave applications have been proposed in earlier studies [12-15]. In fact, most methods of analysis are based on the matrix method [16-20]. Apparently, these methods for designing and simulating layered structures of acoustic wave resonators in software tools are very useful as they allow to determine the optimal structural parameters of resonators [21]. However, with more layers of resonator structure, a large number of linear equations has to be solved.

It is difficult to utilize the general techniques and methods, especially the finite element method (FEM) with the finite structures, multiple units, and complicated boundary conditions. In addition, the earlier matrix formulation and obsolete programming languages may no longer be available 
for more complex calculations or newer piezoelectric devices. Therefore, an approximate method has to be developed to enable accurate analysis of solidly mounted resonators (SMR), i.e., FBAR with the Bragg reflector, or piezoelectric device with new materials. The procedure is very efficient because it requires to predetermine the structure by an analytical method and to use a simulation software before fabrication. Besides, the explicit expressions of vibration frequency and displacement have already been derived by traditional approach, thus one can evaluate with their use the parameters of materials like the density, thickness, and elastic constants directly [22-27]. More rigorous methods and solutions based on the theory for the finite plates of layers can be established with our initial results from the current analysis. It is shown that the essential properties of resonators, such as the vibration frequency, can be accurately obtained. Naturally, it is important to design and optimize resonators by selecting the appropriate materials and optimal thickness of plates.

In this study, we proposed a one-dimensional model of analysis for the thickness-extension mode of FBAR and SMR structures. The onedimensional method for the vibration frequency is applicable for infinite plates, because the width and length of layers are much larger than the thickness, implying a dependence on the thickness only. What is more, the analysis takes into account the coupled interaction between the electrical and mechanical fields to obtain more practical structures in the calculation of the electrical properties of resonators. Finally, it is found that the results of the onedimensional model are reasonably consistent with the results of measurements. The analytical results of frequency have been verified through experimental data of many different types of FBAR with satisfactory agreement. Our research provides new opportunities for producing plate equations of resonators in one-dimensional approach that can provide a better analysis of vibrations of FBAR and SMR devices.

\section{Thickness-extension vibrations of layered thin film plates}

With the assumption that the surface area of plates is infinite due to the finite plate thickness, the vibration equations and solutions automatically become one-dimensional in the analysis. It is generally accepted that using the linear theory of piezoelectricity for layered piezoelectric structures or devices can simplify the analysis of thickness-extension vibrations in the formulation and solution [21-26]. The layered model of a typical piezoelectric resonator is depicted in Fig. 1. The thickness-extension wave is emerged in FBAR device as the functioning vibration modes. The thickness of the $i$-th layer is denoted as $2 b_{i}$, and the origin

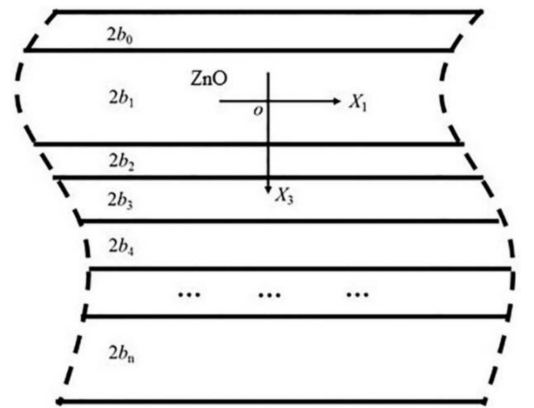

Fig. 1. An FBAR model as a layered structure of thin piezoelectric plates.

of the coordinate system is located in the center of the piezoelectric thin film, as shown in Fig. 1.

The one-dimensional displacements $u_{j}^{(m)}$ of the $m$-th layer $(m=0,1, \ldots, n)$ and electrical potential $\phi$ are assumed as

$$
\begin{aligned}
& u_{1}^{(m)}=u_{2}^{(m)}=0, \\
& u_{3}^{(m)}=u_{3}^{(m)}\left(x_{3}\right) \mathrm{e}^{\mathrm{i} \omega t}, \\
& \phi=\phi\left(x_{3}\right) \mathrm{e}^{\mathrm{i} \omega t},
\end{aligned}
$$

where $x_{3}, \omega, t$, and $n$ are the thickness coordinate, the frequency, the time, and the total number of layers, respectively. The electrical potential is the thickness direction because the piezoelectric thin film is sandwiched by two electrodes.

The strain components of the $m$-th layer with the field variables in (1)-(3) are known and given (we omit the time factor in the abbreviated notions) in the form of [28]:

$$
\begin{aligned}
& S_{1}^{(m)}=S_{2}^{(m)}=S_{4}^{(m)}=S_{5}^{(m)}=S_{6}^{(m)}=0, \\
& S_{3}^{(m)}=\frac{\partial u_{3}^{(m)}}{\partial x_{3}} .
\end{aligned}
$$

For the piezoelectric thin film with the anisotropic materials of $6 \mathrm{~mm}$ crystal family, the nontrivial stresses and electrical displacements, in abbreviated notations, are [28]:

$$
\begin{aligned}
& T_{1}^{(1)}=c_{13}^{(1)} S_{3}^{(1)}+e_{31} \phi_{, 3}, \\
& T_{2}^{(1)}=c_{23}^{(1)} S_{3}^{(1)}+e_{32} \phi_{, 3}, \\
& T_{3}^{(1)}=c_{33}^{(1)} S_{3}^{(1)}+e_{33} \phi_{, 3}=c_{33}^{(1)} u_{3,3}^{(1)}+e_{33} \phi_{, 3}, \\
& D_{3}=e_{33} S_{3}^{(1)}-\varepsilon_{33} \phi_{, 3},
\end{aligned}
$$

where $e_{i p}, c_{p q}$ and $\varepsilon_{i j}$ are the piezoelectric, elastic, and dielectric constants, respectively. A comma followed by an index denotes partial differentiation with respect to the coordinate associated with the index. Here, $\phi_{, 3}=\frac{\partial \phi}{\partial x_{3}}$. The summation convention for repeated indexes is employed.

The stress equation of motion for the layer is then

$$
c_{33}^{(1)} u_{3,33}^{(1)}+e_{33} \phi_{, 33}=-\rho^{(1)} \omega^{2} u_{3}^{(1)},
$$

where $\rho^{(1)}$ is the density of a piezoelectric thin film. A comma followed by two indexes denotes 
second-order partial differentiation with respect to the coordinate associated with the indexes. Here, $\phi_{, 33}=\frac{\partial^{2} \phi}{\partial x_{3}^{2}}$.

$$
D_{1,1}+D_{2,2}+D_{3,3}=e_{33} u_{3,33}^{(1)}-\varepsilon_{33} \phi_{, 33}=0 .
$$

It will yield

$$
\phi=\frac{e_{33}}{\varepsilon_{33}} u_{3}^{(1)}+C_{1} x_{3}+C_{2},
$$

where $C_{1}$ and $C_{2}$ are the two constants of integration.

A substitution of the electrical potential solution (12) into the constitutive relations (6)-(9), will yield

$$
\begin{aligned}
& T_{3}^{(1)}=\bar{c}_{33}^{(1)} u_{3,3}^{(1)}+e_{33} C_{1}, \\
& D_{3}=-\varepsilon_{33} C_{1} .
\end{aligned}
$$

The displacement equation of motion (10) will be

$$
\bar{c}_{33}^{(1)} u_{3,33}^{(1)}=-\rho^{(1)} \omega^{2} u_{3}^{(1)},
$$

where

$$
\bar{c}_{33}^{(1)}=c_{33}^{(1)}\left(1+\frac{e_{33}^{2}}{c_{33}^{(1)} \varepsilon_{33}}\right)
$$

is the piezoelectrically stiffened elastic constant. We can assume the solution (15) of displacement as

$$
u_{3}^{(1)}=A^{(1)} \sin \left(\xi^{(1)} x_{3}\right)+B^{(1)} \cos \left(\xi^{(1)} x_{3}\right),
$$

where $\xi^{(1)}$ is the wave number of a piezoelectric thin film.

By substituting (17) into (15), one yields the frequency solution, i.e.,

$$
\omega^{2}=\frac{\bar{c}_{33}^{(1)}}{\rho^{(1)}}\left(\xi^{(1)}\right)^{2} .
$$

Then, the electrical potential and stress solutions in the piezoelectric thin film will be

$$
\begin{aligned}
& \phi= \\
& \quad+\frac{e_{33}}{\varepsilon_{33}}\left[A^{(1)} \sin \left(\xi^{(1)} x_{3}\right)+B^{(1)} \cos \left(\xi^{(1)} x_{3}\right)\right] \\
& +C_{1} x_{3}+C_{2}, \\
& T_{3}^{(1)}=\bar{c}_{33}^{(1)} \xi^{(1)}\left[A^{(1)} \cos \left(\xi^{(1)} x_{3}\right)\right. \\
& \left.\quad-B^{(1)} \sin \left(\xi^{(1)} x_{3}\right)\right]+e_{33} C_{1} .
\end{aligned}
$$

With a constant alternating electric field on the electrodes, it follows that

$$
\phi\left( \pm b_{1}\right)= \pm \phi_{0} \text {. }
$$

Applying (21) to (19) and (20), we now have

$$
\begin{aligned}
C_{1} & =\frac{\phi_{0}}{b_{1}}-\frac{e_{33}}{\varepsilon_{33}} \frac{A^{(1)} \sin \left(\xi^{(1)} b_{1}\right)}{b_{1}}, \\
C_{2} & =-\frac{e_{33}}{\varepsilon_{33}} B^{(1)} \cos \left(\xi^{(1)} b_{1}\right), \\
T_{3}^{(1)} & = \\
A^{(1)} & {\left[\bar{c}_{33}^{(1)} \xi^{(1)} \cos \left(\xi^{(1)} x_{3}\right)-\frac{e_{33}^{2}}{\varepsilon_{33}} \frac{\sin \left(\xi^{(1)} b_{1}\right)}{b_{1}}\right] } \\
- & B^{(1)} \bar{c}_{33}^{(1)} \xi^{(1)} \sin \left(\xi^{(1)} x_{3}\right)+\frac{e_{33}}{b_{1}} \phi_{0} .
\end{aligned}
$$

Note that there is no need to consider the driving voltage on the Bragg reflector and substrate layer. As a result, only the mechanical displacement will enter into the equations of the nonpiezoelectric thin film with

$$
S_{3}^{(m)}=\frac{\partial u_{3}^{(m)}}{\partial x_{3}}
$$

for $m=0,2,3, \ldots, n$. The constitutive relations will be

$$
\begin{aligned}
& T_{1}^{(m)}=c_{13}^{(m)} S_{3}^{(m)}, \\
& T_{2}^{(m)}=c_{23}^{(m)} S_{3}^{(m)}, \\
& T_{3}^{(m)}=c_{33}^{(m)} S_{3}^{(m)} .
\end{aligned}
$$

The displacement equations of motion will give us

$$
c_{33}^{(m)} \frac{\partial^{2} u_{3}^{(m)}}{\partial x_{3}^{2}}=-\rho^{(m)} \omega^{2} u_{3}^{(m)},
$$

while the displacement solution of a nonpiezoelectric layer will be

$$
\begin{gathered}
u_{3}^{(m)}\left(x_{3}\right)=A^{(m)} \sin \left(\xi^{(m)} x_{3}\right) \\
+B^{(m)} \cos \left(\xi^{(m)} x_{3}\right) .
\end{gathered}
$$

The frequency solution will yield

$$
\omega^{2}=\frac{c_{33}^{(m)}}{\rho^{(m)}}\left(\xi^{(m)}\right)^{2} .
$$

The wave numbers will be related to each other through

$$
\xi^{(m)}=\sqrt{\frac{\bar{c}_{33}^{(1)} \rho^{(m)}}{c_{33}^{(m)} \rho^{(1)}}} \xi^{(1)} .
$$

Finally, the stress solutions of a nonpiezoelectric layer will be given as

$$
\begin{aligned}
& T_{3}^{(m)}=c_{33}^{(m)} \xi^{(m)} \\
& \quad \times\left[A^{(m)} \cos \left(\xi^{(m)} x_{3}\right)-B^{(m)} \sin \left(\xi^{(m)} x_{3}\right)\right] .
\end{aligned}
$$

Since the electrical potential has been obtained and incorporated into the stress equations, the tractionfree and continuity conditions become as follows

$$
\begin{aligned}
& T_{3}^{(0)}\left(-b_{1}-2 b_{0}\right)=0, \\
& T_{3}^{(n)}\left(h_{n}\right)=0, \\
& T_{3}^{(m)}\left(h_{m}\right)=T_{3}^{(m+1)}\left(h_{m}\right), \\
& u_{3}^{(m)}\left(h_{m}\right)=u_{3}^{(m+1)}\left(h_{m}\right),
\end{aligned}
$$

where

$$
\begin{aligned}
& h_{0}=-b_{1}, \\
& h_{m}=-b_{1}+2 \sum_{m}^{n} b_{m}, \quad m=1, \ldots, n-1 .
\end{aligned}
$$

Through the substitution of the stress equations (22)-(24) and (33) into the boundary conditions (34)-(37), the boundary conditions will be transformed to 


$$
\begin{aligned}
& A^{(0)} \cos \left(\xi^{(0)}\left(-b_{1}-2 b_{0}\right)\right)-B^{(0)} \sin \left(\xi^{(0)}\left(-b_{1}-2 b_{0}\right)\right)=0 \\
& A^{(n)} \cos \left(\xi^{(n)} h_{n}\right)-B^{(n)} \sin \left(\xi^{(n)} h_{n}\right)=0 \\
& c_{33}^{(0)} \xi^{(0)}\left[A^{(0)} \cos \left(\xi^{(0)} b_{1}\right)+B^{(0)} \sin \left(\xi^{(0)} b_{1}\right)\right]-A^{(1)}\left[\bar{c}_{33}^{(1)} \xi^{(1)} \cos \left(\xi^{(1)} b_{1}\right)-\frac{e_{33}^{2}}{\varepsilon_{33}} \frac{\sin \left(\xi^{(1)} b_{1}\right)}{b_{1}}\right] \\
& -B^{(1)} \bar{c}_{33}^{(1)} \xi^{(1)} \sin \left(\xi^{(1)} b_{1}\right)=\frac{e_{33}}{b_{1}} \phi_{0}, \\
& -A^{(0)} \sin \left(\xi^{(0)} b_{1}\right)+B^{(0)} \cos \left(\xi^{(0)} b_{1}\right)+A^{(1)} \sin \left(\xi^{(1)} b_{1}\right)-B^{(1)} \cos \left(\xi^{(1)} b_{1}\right)=0 \\
& A^{(1)}\left[\bar{c}_{33}^{(1)} \xi^{(1)} \cos \left(\xi^{(1)} b_{1}\right)-\frac{e_{33}^{2}}{\varepsilon_{33}} \frac{\sin \left(\xi^{(1)} b_{1}\right)}{b_{1}}\right]-B^{(1)} \bar{c}_{33}^{(1)} \xi^{(1)} \sin \left(\xi^{(1)} b_{1}\right) \\
& -c_{33}^{(2)} \xi^{(2)}\left[A^{(2)} \cos \left(\xi^{(2)} b_{1}\right)-B^{(2)} \sin \left(\xi^{(2)} b_{1}\right)\right]=-\frac{e_{33}}{b_{1}} \phi_{0}, \\
& A^{(1)} \sin \left(\xi^{(1)} b_{1}\right)+B^{(1)} \cos \left(\xi^{(1)} b_{1}\right)-A^{(2)} \sin \left(\xi^{(2)} b_{1}\right)-B^{(2)} \cos \left(\xi^{(2)} b_{1}\right)=0 \\
& A_{33}^{(m)} \xi^{(m)}\left[A^{(m)} \cos \left(\xi^{(m)} h_{m}\right)-B^{(m)} \sin \left(\xi^{(m)} h_{m}\right)\right] \\
& -c_{33}^{(m+1)} \xi^{(m+1)}\left[A^{(m+1)} \cos \left(\xi^{(m+1)} h_{m}\right)-B^{(m+1)} \sin \left(\xi^{(m+1)} h_{m}\right)\right]=0 \\
& \left.A_{m}\right)+B^{(m)} \cos \left(\xi^{(m)} h_{m}\right)-A^{(m+1)} \sin \left(\xi^{(m+1)} h_{m}\right)-B^{(m+1)} \cos \left(\xi^{(m+1)} h_{m}\right)=0
\end{aligned}
$$

where $m=1,2,3, \ldots, n-1$.

The displacement solutions could be solved in terms of the amplitudes of each layer using (40)-(47) and with given driving voltage $\phi_{0}$.

In this simplified model, the displacement solutions have very limited applications. In fact, even the displacements themselves are not actually needed for circuit applications of a resonator. We need to obtain the solutions for the design and optimization of FBAR and SMR structures. The available solution here is the resonant frequency that is related to the material properties and thicknesses of layers, which are actually the essential parameters of a resonator structure. The adequately selected materials and thicknesses will not only determine the vibration frequency but also change the device properties. As a result, a careful study of the vibration frequency in terms of the material properties and layer thickness will be important in the prototype and improvement process of resonators.

The boundary condition equations in (40)-(47) are no longer the homogeneous equations for the calculation of natural frequency. They have a driving voltage term in the right-hand side of the equations, implying this is a forced vibration problem. However, Tiersten et al. [29] demonstrated that the resonant frequency of a device can be obtained as setting the coefficient determinant to vanish. The frequency equation which depends on the quantity of layers is usually a transcendental equation, and it must be solved numerically. We presented a few cases of FBAR structures with a smaller number of layers, and compared them with earlier equations of the mechanical vibrations in order to explore the coupling and effect of piezoelectric properties in the frequency equation.

For example, for one-layer structures of FBAR, the frequency equation is [22]:

$$
\tan \left(\xi^{(1)} b_{1}\right)=\frac{\bar{c}_{33}^{(1)} \xi^{(1)} \varepsilon_{33} b_{1}}{e_{33}^{2}} .
$$

In turn, for two-layer structures, the frequency equation takes the form of [22]:

$$
\begin{aligned}
& {\left[1-\frac{e_{33}^{2}}{\varepsilon_{33} \bar{c}_{33}^{(1)} \xi^{(1)} b_{1}} \tan \left(\xi^{(1)} b_{1}\right)\right]} \\
& \quad \times\left[\frac{c_{33}^{(0)} \xi^{(0)}}{\bar{c}_{33}^{(1)} \xi^{(1)}} \tan \left(2 \xi^{(0)} b_{0}\right)+2 \tan \left(\xi^{(1)} b_{1}\right)\right]= \\
& \quad \frac{c_{33}^{(0)} \xi^{(0)}}{\bar{c}_{33}^{(1)} \xi^{(1)}} \tan \left(2 \xi^{(0)} b_{0}\right) \tan ^{2}\left(\xi^{(1)} b_{1}\right) .
\end{aligned}
$$

For three-layer structures of FBAR, the frequency equation is $[22,23]$ : 


$$
\begin{aligned}
& \left(1-\frac{e_{33}^{2}}{\varepsilon_{33} \bar{c}_{33}^{(1)} \xi^{(1)} b_{1}} \tan \left(\xi^{(1)} b_{1}\right)\right)\left[\frac{c_{33}^{(0)} \xi^{(0)}}{\bar{c}_{33}^{(1)} \xi^{(1)}} \tan \left(2 \xi^{(0)} b_{0}\right)+2 \tan \left(\xi^{(1)} b_{1}\right)+\frac{c_{33}^{(2)} \xi^{(2)}}{\bar{c}_{33}^{(1)} \xi^{(1)}} \tan \left(2 \xi^{(2)} b_{2}\right)\right]= \\
& \tan \left(\xi^{(1)} b_{1}\right)\left\{\tan \left(\xi^{(1)} b_{1}\right)\left[\frac{c_{33}^{(0)} \xi^{(0)}}{\bar{c}_{33}^{(1)} \xi^{(1)}} \tan \left(2 \xi^{(0)} b_{0}\right)+\frac{c_{33}^{(2)} \xi^{(2)}}{\bar{c}_{33}^{(1)} \xi^{(1)}} \tan \left(2 \xi^{(2)} b_{2}\right)\right]\right. \\
& \left.\quad+2 \frac{c_{33}^{(0)} \xi^{(0)} c_{33}^{(2)} \xi^{(2)}}{\left[\bar{c}_{33}^{(1)} \xi^{(1)}\right]^{2}} \tan \left(2 \xi^{(0)} b_{0}\right) \tan \left(2 \xi^{(2)} b_{2}\right)\right\} .
\end{aligned}
$$

For the case with an FBAR structure over three layers, the transcendental equations will be too complex to present in explicit forms. However, it could be feasible to construct the equation by utilizing the known pattern of the determinant, because the frequency equations are obtained from the coefficient determinant of the boundary condition equations. In fact, the vibration frequency of multilayered structure devices can also be obtained in this way. The general structure of the determinant will be

$$
\left[\begin{array}{ccccccccccccc}
A_{11} & A_{12} & 0 & 0 & 0 & 0 & \cdots & 0 & 0 & 0 & 0 & 0 & 0 \\
0 & 0 & 0 & 0 & 0 & 0 & \cdots & 0 & 0 & 0 & 0 & A_{2, n-1} & A_{2, n} \\
A_{31} & A_{32} & A_{33} & A_{34} & 0 & 0 & \cdots & 0 & 0 & 0 & 0 & 0 & 0 \\
A_{41} & A_{42} & A_{43} & A_{44} & 0 & 0 & \cdots & 0 & 0 & 0 & 0 & 0 & 0 \\
0 & 0 & A_{53} & A_{54} & A_{55} & A_{56} & \cdots & 0 & 0 & 0 & 0 & 0 & 0 \\
0 & 0 & A_{63} & A_{64} & A_{65} & A_{66} & \cdots & 0 & 0 & 0 & 0 & 0 & 0 \\
\vdots & \vdots & \vdots & \vdots & \vdots & \vdots & \ddots & \vdots & \vdots & \vdots & \vdots & \vdots & \vdots \\
0 & 0 & 0 & 0 & 0 & 0 & \cdots & A_{n-3, n-5} & A_{n-3, n-4} & A_{n-3, n-3} & A_{n-3, n-2} & 0 & 0 \\
0 & 0 & 0 & 0 & 0 & 0 & \cdots & A_{n-2, n-5} & A_{n-2, n-4} & A_{n-2, n-3} & A_{n-2, n-2} & 0 & 0 \\
0 & 0 & 0 & 0 & 0 & 0 & \cdots & 0 & 0 & A_{n-1, n-3} & A_{n-1, n-2} & A_{n-1, n-1} & A_{n-1, n} \\
0 & 0 & 0 & 0 & 0 & 0 & \cdots & 0 & 0 & A_{n, n-3} & A_{n, n-2} & A_{n, n-1} & A_{n, n}
\end{array}\right]
$$

where

$$
\begin{aligned}
& A_{11}=\cos \left(\xi^{(0)}\left(-b_{1}-2 b_{0}\right)\right) \\
& A_{12}=-\sin \left(\xi^{(0)}\left(-b_{1}-2 b_{0}\right)\right) \\
& A_{2, n-1}=\cos \left(\xi^{(n)} h_{n}\right) \\
& A_{2, n}=-\sin \left(\xi^{(n)} h_{n}\right) \\
& A_{31}=c_{33}^{(0)} \xi^{(0)} \cos \left(\xi^{(0)} b_{1}\right) \\
& A_{32}=c_{33}^{(0)} \xi^{(0)} \sin \left(\xi^{(0)} b_{1}\right) \\
& A_{33}=-\bar{c}_{33}^{(1)} \xi^{(1)} \cos \left(\xi^{(1)} b_{1}\right)+\frac{e_{33}^{2}}{\varepsilon_{33}} \frac{\sin \left(\xi^{(1)} b_{1}\right)}{b_{1}} \\
& A_{34}=-\bar{c}_{33}^{(1)} \xi^{(1)} \sin \left(\xi^{(1)} b_{1}\right) \\
& A_{41}=-\sin \left(\xi^{(0)} b_{1}\right)
\end{aligned}
$$

$$
\begin{aligned}
& A_{42}=\cos \left(\xi^{(0)} b_{1}\right) \\
& A_{43}=\sin \left(\xi^{(1)} b_{1}\right) \\
& A_{44}=-\cos \left(\xi^{(1)} b_{1}\right) \\
& A_{53}=\bar{c}_{33}^{(1)} \xi^{(1)} \cos \left(\xi^{(1)} b_{1}\right)-\frac{e_{33}^{2}}{\varepsilon_{33}} \frac{\sin \left(\xi^{(1)} b_{1}\right)}{b_{1}} \\
& A_{54}=-\bar{c}_{33}^{(1)} \xi^{(1)} \sin \left(\xi^{(1)} b_{1}\right) \\
& A_{55}=-c_{33}^{(2)} \xi^{(2)} \cos \left(\xi^{(2)} b_{1}\right) \\
& A_{56}=c_{33}^{(2)} \xi^{(2)} \sin \left(\xi^{(2)} b_{1}\right) \\
& A_{63}=\sin \left(\xi^{(1)} b_{1}\right) \\
& A_{64}=\cos \left(\xi^{(1)} b_{1}\right)
\end{aligned}
$$




$$
\begin{aligned}
& A_{65}=-\sin \left(\xi^{(2)} b_{1}\right) \\
& A_{66}=-\cos \left(\xi^{(2)} b_{1}\right) \\
& A_{n-3, n-5}=c_{33}^{(n-2)} \xi^{(n-2)} \cos \left(\xi^{(n-2)} h_{n-2}\right) \text {, } \\
& A_{n-3, n-4}=-c_{33}^{(n-2)} \xi^{(n-2)} \sin \left(\xi^{(n-2)} h_{n-2}\right), \\
& A_{n-3, n-3}=-c_{33}^{(n-1)} \xi^{(n-1)} \cos \left(\xi^{(n-1)} h_{n-2}\right), \\
& A_{n-3, n-2}=c_{33}^{(n-1)} \xi^{(n-1)} \sin \left(\xi^{(n-1)} h_{n-2}\right), \\
& A_{n-2, n-5}=\sin \left(\xi^{(n-2)} h_{n-2}\right), \\
& A_{n-2, n-4}=\cos \left(\xi^{(n-2)} h_{n-2}\right), \\
& A_{n-2, n-3}=-\sin \left(\xi^{(n-1)} h_{n-2}\right), \\
& A_{n-2, n-2}=-\cos \left(\xi^{(n-1)} h_{n-2}\right), \\
& A_{n-1, n-3}=c_{33}^{(n-1)} \xi^{(n-1)} \cos \left(\xi^{(n-1)} h_{n-1}\right), \\
& A_{n-1, n-2}=-c_{33}^{(n-1)} \xi^{(n-1)} \sin \left(\xi^{(n-1)} h_{n-1}\right), \\
& A_{n-1, n-1}=-c_{33}^{(n)} \xi^{(n)} \cos \left(\xi^{(n)} h_{n-1}\right) \\
& A_{n-1, n}=c_{33}^{(n)} \xi^{(n)} \sin \left(\xi^{(n)} h_{n-1}\right), \\
& A_{n, n-3}=\sin \left(\xi^{(n-1)} h_{n-1}\right) \\
& A_{n, n-2}=\cos \left(\xi^{(n-1)} h_{n-1}\right), \\
& A_{n, n-1}=-\sin \left(\xi^{(n)} h_{n-1}\right), \\
& A_{n, n}=-\cos \left(\xi^{(n)} h_{n-1}\right)
\end{aligned}
$$

Recapitulating, the determinant of a resonator structure with a given number of layers will be constructed with patterned components which are listed above. Moreover, the determinant for frequency and vibration solutions could be evaluated by utilizing the available numerical techniques without difficulties. These results are certainly useful for the analysis of structures with a larger number of layers.

\section{Numerical examples}

The frequency equations and displacement solutions shown in Sect. 2 which can be utilized to explore the thickness-extension vibrations of layered structures, such as typical FBARs and SMRs, are

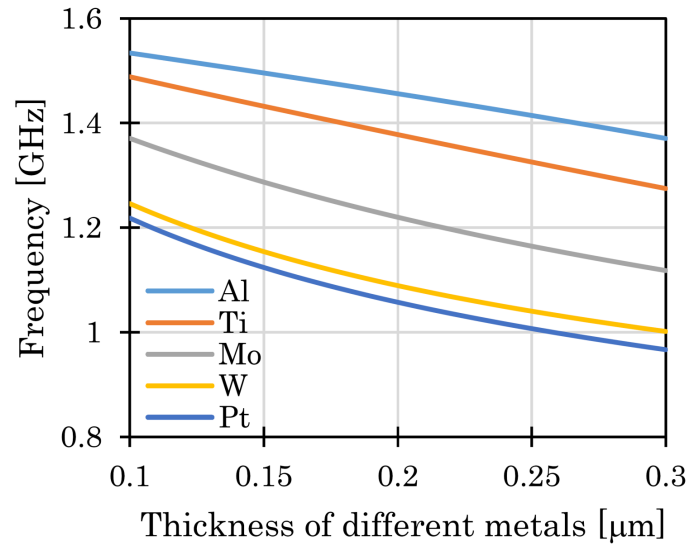

Fig. 2. Frequency dependence of a three-layer thin film structure on the thickness of symmetric metal electrodes.

TABLE I

Calculated thickness-extension frequencies of a layered structure with different layer thicknesses in comparison with measurement.

\begin{tabular}{c|c|c|c|c|c}
\hline $\begin{array}{c}\text { Bottom } \\
\text { electrode } \\
\mathrm{Al}[\mu \mathrm{m}]\end{array}$ & $\begin{array}{c}\text { Top } \\
\text { electrode } \\
\mathrm{Al}[\mu \mathrm{m}]\end{array}$ & $\begin{array}{c}\mathrm{ZnO} \\
{[\mu \mathrm{m}]}\end{array}$ & $\begin{array}{c}\mathrm{Si}_{3} \mathrm{~N}_{4} \\
{[\mu \mathrm{m}]}\end{array}$ & $\begin{array}{c}\text { Measurement } \\
{[\mathrm{GHz}]} \\
\text { Ref. [30] }\end{array}$ & $\begin{array}{c}\text { Calculation } \\
{[\mathrm{GHz}]}\end{array}$ \\
\hline 0.1 & 0.1 & 2 & 0.9 & 1.2 & 1.1600 \\
0.2 & 0.2 & 2 & 0.2 & 1.4 & 1.3485 \\
0.1 & 0.1 & 1 & 0.2 & 2.5 & 2.4752
\end{tabular}

presented and compared with earlier results from both mechanical and piezoelectric vibrations. We want to perform some calculations with the procedure so that comparisons and verifications can be demonstrated.

\subsection{Example 1}

The four-layer FBAR structure consists of top and bottom aluminum (Al) electrodes, zinc oxide $(\mathrm{ZnO})$ piezoelectric thin film, and silicon nitride $\left(\mathrm{Si}_{3} \mathrm{~N}_{4}\right)$ substrate. Table I lists the results from the thickness-extension vibrations. These are considerably close to the actual measurement of resonant frequency from the actual fabricated resonators [30]. The errors frequencies of the three structures studied in this research are $3.33 \%, 3.68 \%$ and $0.99 \%$, respectively.

\subsection{Example 2}

The piezoelectric effect for the vibration frequency is our major concern in this study. In order to test the effect of piezoelectric stiffening with different metal electrodes and thickness, we calculated a three-layer FBAR structure with symmetric electrodes and the thickness of a $\mathrm{ZnO}$ layer of $2 \mu \mathrm{m}$ vibrating at the thickness-extension mode. The significant changes of frequencies in the thin film structure are shown in Fig. 2. It is clear that the vibration frequency decreased with the increase of 


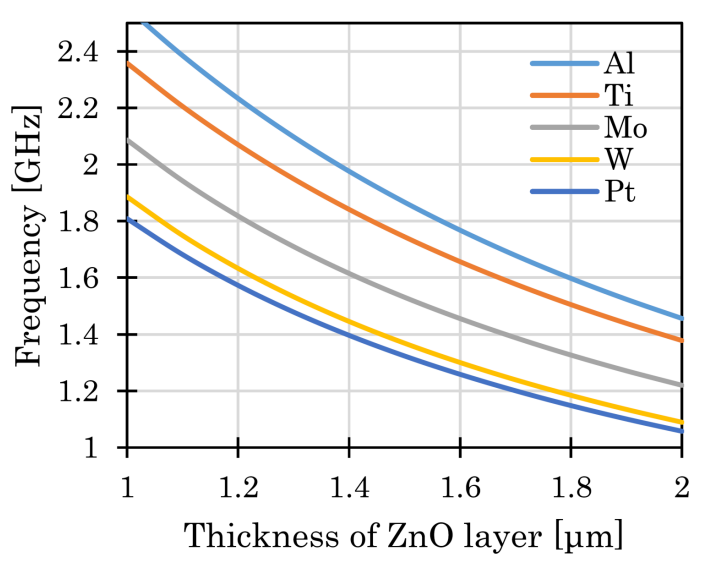

Fig. 3. Frequency dependence on the thickness of a $\mathrm{ZnO}$ piezoelectric layer in a three-layer structure with the thickness of electrodes of $200 \mathrm{~nm}$.

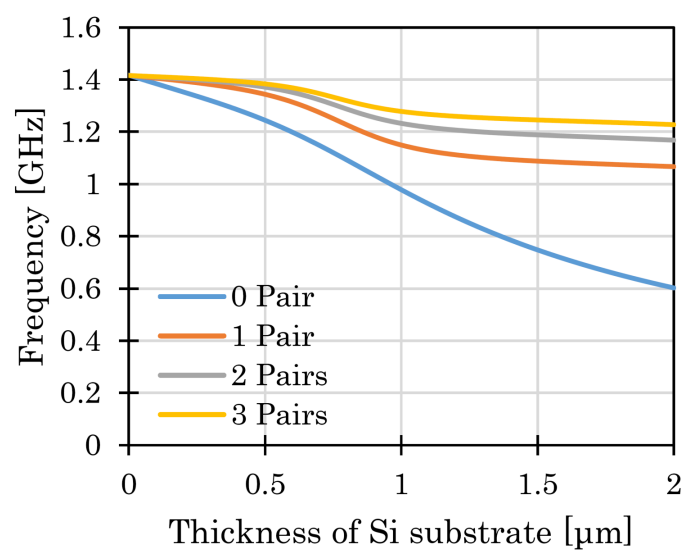

Fig. 4. Frequency dependence on the thickness of a silicon substrate for a multiple-layer structure in the thickness-extension mode.

thickness of electrodes because of mass loading. Besides, different materials of electrodes with the same thickness result in different shifts of vibration frequency, because the higher the density of materials, the more gravimetric loading on the FBAR.

\subsection{Example 3}

Figure 3 depicts the thickness-extension variation frequency of a three-layer FBAR structure, which consists of fixed $200 \mathrm{~nm}$ electrodes and the variation of the thickness of a $\mathrm{ZnO}$ piezoelectric thin film. The results are the same as the last example where the resonant frequencies are significantly decreased with the increase of the thickness of layers.

\subsection{Example 4}

Figure 4 depicts the thickness-extension frequency of a ZnO-based FBAR with a Bragg reflector. The piezoelectric element consists of a $480 \mathrm{~nm}$ $\mathrm{ZnO}$ piezoelectric thin film and $100 \mathrm{~nm}$ top and bottom Pt electrodes. The Bragg reflector consists of periodic high and low impedance layers. The low impedance layers are $\mathrm{ZnO}$ of $1080 \mathrm{~nm}$ and the high impedance layers use Pt with $710 \mathrm{~nm}$. The initial resonance frequencies of $\mathrm{FBAR}$ with different pairs of the Bragg reflection layers are same. It is interesting to find out that the vibration frequencies have certain regions with relatively flat curves at certain thickness of the substrate, implying design windows for a possible insensitive selection of substrate thickness. We also found that the distance between the Bragg reflection layers becomes smaller in this case. It means the majority of energy of acoustic waves is limited in the piezoelectric element. Therefore, our results are very reasonable for the purpose of the Bragg reflection layers' design.

\section{Conclusions}

From the vibration analysis of multi-layered structures representing the FBAR with the consideration of piezoelectric properties, we have obtained accurate resonant frequencies of these stacked layers vibrating in the thickness-extension mode. By comparing the analytical results with known measurements from actual prototypes of FBAR resonators, we have proven that the analytical procedure based on the layered plate model can provide accurate prediction of the vibration frequency which is the essential parameter of resonators. Furthermore, the effect of the Bragg reflectors in FBAR could be deduced by this analysis. Such a simple model also proves that in the FBAR structures, due to the relatively larger size in the plane, the vibration is almost solely determined by the sequence and thickness of each layer. In other words, the effect of plane sizes has limited influence on the vibration frequencies. This signifies the thickness vibrations of a layered high-frequency resonator structure, and the thickness deformation is dominant in the plate plane. In this case, an approximate analysis with thickness deformation should provide a reasonable estimation of resonator properties in addition to the vibration frequency shown in this study. Or, the approximation of other field variables should also be made as demonstrated with the calculation of the functioning frequency. It also suggests that in the design process, we can use the one-dimensional method presented in this study to calculate the vibration frequency and the resonator development should be focused on the electrical properties, which may be better predicted based on the structural patterns and interactions of different partitions and layouts.

The future development may go in two directions in the analysis and modeling through the frequency determination with one-dimensional structures with all layers and the electrical properties which are obtained from the patterns of electrodes and layout of conducting and piezoelectric materials in the plane. Of course, we need further studies on vibration analysis to verify the accuracy of vibration frequencies of the structures, acoustic wave field, and distribution of electrical field for the estimation of other parameters and properties. 


\section{Acknowledgments}

This research was supported in part by the Technology Innovation 2025 Program of Municipality of Ningbo (Grant No 2019B10122) and the Research and Development Program of Key Disciplines of Guangdong Province (Grant 2020B0101040002).

\section{References}

[1] G. Zeng, Ch. Wu, Y. Chang, Ch. Zhou, B. Chen, M. Zhang, J. Li, X. Duan, Q. Yang, W. Pang, ACS Sensors 4, 1524 (2019).

[2] Z.X. Zhang, J. Liang, D.H. Zhang, W. Pang, H. Zhang, Micromachines 6, 1306 (2015).

[3] R. Aigner, in: 13th Int. Conf. on SolidState Sensors, Actuators and Microsystems, Digest of Technical Papers, TRANSDUCERS'05, 2005, p. 5.

[4] R.C. Lu, T. Manzaneque, Y.S. Yang, J. Zhou, H. Hassanieh, S.B. Gong, J. Microelectromech. Systems 27, 931 (2018).

[5] E. Milyutin, P. Muralt, IEEE Trans. Ultrason. Ferroelectr. Freq. Control. 58, 685 (2011).

[6] J. Filipiak, G. Steczko, S. Kostrzewa, L. Wcislo, Acta Phys. Pol. A 128, 408 (2015).

[7] J. Filipiak, L. Solarz, G. Steczko, Acta Phys. Pol. A 120, 593 (2011).

[8] I.V. Kubasov, A.M. Kislyuk, M.D. Malinkovich, A.A. Temirov, S.V. Ksenich, D.A. Kiselev, A.S. Bykov, Y.N. Parkhomenko, Acta Phys. Pol. A 134, 106 (2018).

[9] H.H. Guo, A.H. Guo, Y. Gao, T.T. Liu, Instrum. Sci. Technol. 48, 431 (2020).

[10] R. Ruby, in: 2007 IEEE Ultrasonics Symp. Proc., 2007, p. 1029.

[11] R. Ruby, K. Sankaragomathi, S. Sridaran, R. Parker, in: 2015 IEEE Int. Ultrasonics Symp., 2015.

[12] W.M. Ewing, W.S. Jardetzky, F. Press, Elastic Wave in Layered Media, McGrawHill, New York 1957.
[13] L.M. Brekhovskikh, O.A. Godin, Acoustics of Layered Media I: Plane and Quasi-Plane Waves, Springer-Verlag, Berlin 1990.

[14] L.M. Brekhovskikh, O.A. Godin, Acoustics of Layered Media II: Point Sources and Bounded Beams, Springer, Berlin 1992.

[15] L.F. Qin, Q.M. Chen, H.B. Cheng, Q.M. Wang, IEEE Trans. Ultrason. Ferroelectr. Freq. Control. 57, 1840 (2010).

[16] E.L. Adler, IEEE Trans. Ultrason. Ferroelectr. Freq. Control. 37, 485 (1990).

[17] J.T. Stewart, Y.K. Yong, IEEE Trans. Ultrason. Ferroelectr. Freq. Control. 41, 375 (1994).

[18] W.Q. Chen, Y.Q. Guo, in: 2007 IEEE Ultrasonics Sympos. Proc., 2007, p. 2307.

[19] V.Y. Zhang, B. Dubus, B. Collet, M. Destrade, J. Acoust. Soc. Am. 123, 1972 (2008).

[20] Y.Q. Guo, W.Q. Chen, Y.L. Zhang, Sci. China Ser. G 52, 1094 (2009).

[21] E.L. Adler, IEEE Trans. Ultrason. Ferroelectr. Freq. Control. 37, 485 (1990).

[22] J.S. Yang, H.G. Zhou, W.P. Zhang, IEEE Trans. Ultrason. Ferroelectr. Freq. Control. 52, 918 (2005).

[23] J. Wang, L.J. Shen, J. Zhejiang Univ. Sci. A 6, 980 (2005).

[24] J. Wang, J.S. Liu, J.K. Du, D.J. Huang, in: 2008 Symp. on Piezoelectricity, Acoustic Waves, and Device Applications, 2008, p. 72 .

[25] J. Wang, L.J. Shen, J.S. Yang, Ultrasonics 48, 150 (2008).

[26] D.J. Huang, J. Wang, J.K. Du, in: 2008 IEEE Int. Frequency Control Symp., 2008, p. 204.

[27] J.K. Du, K. Xian, J. Wang, J.S. Yang, Ultrasonics 49, 149 (2009).

[28] H. F. Tiersten, Linear Piezoelectric Plate Vibrations, Springer, Boston (MA) 1969.

[29] J.L. Bleustein, H.F. Tiersten, J. Acoust. Soc. Am. 43, 1311 (1968).

[30] H. Zhang, W. Pang, E.S. Kim, in: Proc. 2005 IEEE Int. Frequency Control Symp. and Exposition, 2005, p. 73. 